\title{
Immunocytochemical Characterization of Murine Hex, a Homeobox-Containing Protein
}

\author{
BIDYUT GHOSH, GHEORGHE R. GANEA, LEE A. DENSON, ROCCO IANNUCCI, \\ HARRIS C. JACOBS, AND CLIFFORD W. BOGUE
}

\begin{abstract}
Department of Pediatrics, Yale University School of Medicine, New Haven, Connecticut 06520, U.S.A. [B.G., G.R.G., L.A.D., R.I., C.W.B.]; and Department of Pediatrics, Bridgeport Hospital, Bridgeport, Connecticut 06610, U.S.A. [H.C.J.]
\end{abstract}

\begin{abstract}
A polyclonal antibody against a glutathione $S$-transferase fusion protein containing the $76 \mathrm{COOH}$-terminal amino acids of Hex, a divergent homeobox gene, was raised in rabbits. Western blot and immunofluorescence reveal that Hex is a $35-37-\mathrm{kD}$ soluble protein present both in the nucleus and cytoplasm of transfected and nontransfected cultured cells as well as in whole mouse embryo. Confocal microscopy of whole mount immunostained mouse embryos at E7.5 and E8.5 demonstrates that Hex is differentially localized in the cytoplasm and nucleus of definitive endoderm, developing blood islands, and hepatic diverticulum. In particular, in the region of the foregut that gives rise to the liver, Hex expression is nuclear in the endodermal cells of the hepatic diverticulum, whereas expression is primarily cytoplas-
\end{abstract}

\section{ABSTRACT}

mic in cells lateral to the liver-forming region. This suggests that nuclear localization of Hex is involved in early hepatic specification and that compartmentalization of Hex protein plays an important role in its function during mouse development. (Pediatr Res 48: 634-638, 2000)

\[ \begin{array}{c}\text { Abbreviations } \\ \text { GST, glutathione } S \text {-transferase }\end{array} \]
IPTG, isopropyl $\beta$-D-thiogalactopyranoside
PMSF, phenylmethylsulfonyl fluoride
PBST, 0.1\% Triton X-100 in PBS
NLS, nuclear localization signal
NES, nuclear export signal

Homeobox gene products are transcription factors containing a conserved 60-amino acid homeodomain that binds DNA in a sequence-specific manner and regulates different aspects of cell differentiation and morphogenesis during animal development $(1,2)$. The divergent or orphan homeobox gene Hex (3), also known as Prh (4), is expressed in a variety of multipotent hematopoietic progenitor cells and cell lines, suggesting a role in the early stages of hematopoietic cell differentiation. Recently Hex has been implicated as the earliest unequivocal molecular marker of anteroposterior patterning in the mouse embryo (5) and is also believed to play a role in organogenesis on the basis of its expression in the developing liver, thyroid, and lung (6).

In general, the expression patterns of homeobox genes, including Hex, have mainly been examined at the mRNA level by use of in situ hybridization or RNase protection assay. However, the expression patterns of homeobox mRNA and their proteins do not always correlate, possibly because of

Received March 24, 2000; accepted June 20, 2000

Correspondence: Clifford W. Bogue, M.D., Section of Critical Care and Applied Physiology, Department of Pediatrics, Yale University School of Medicine, PO Box 208064, 333 Cedar Street, New Haven, CT 06520-8064, U.S.A.

Supported by NIH Training Grant T32 HL07272 (B.G.), NIH K08 HL03471 (C.W.B.), and Yale Liver Center Pilot grant (H.C.J. and C.W.B.). posttranscriptional and/or posttranslational modifications (7, 8). For example, maternally derived caudal mRNA is distributed diffusely but its protein is expressed in a gradient fashion only in the posterior area of the Drosophila embryo (9), and $C s x / N k x \quad 2.5$ mRNA is readily detectable in National Institutes of Health 3 T3 cells but its protein product is undetectable (10). Another level of homeobox gene regulation involves the spatial distribution of its protein product within the cell. The Exd product accumulates only in the cell cytoplasm in early embryos and is later translocated to the nucleus $(11,12)$. Endogenous rat engrailed homeoproteins are present in the cytosolic and membrane fractions in addition to their nuclear localization in embryonic midbrain/hindbrain (13). Although a considerable amount of information regarding the distribution and expression pattern of Hex is available at the mRNA level in vertebrate development $(3,5,6,14,15)$, nothing is known about the localization of the Hex protein.

The purpose of the present study was to identify and localize Hex protein at the cellular and subcellular levels in transfected and nontransfected cells as well as in whole mouse embryos by use of a polyclonal antibody. We found that Hex is a soluble protein with an apparent molecular mass of 35-37 kD. Surprisingly, it is localized both in the nucleus and cytoplasm of transfected COS cells and nontransfected MLE-15 cells. We 
also show, by whole mount immunostaining of mouse embryos, that Hex is expressed in the definitive endoderm and blood islands at E7.5 and in the hepatic diverticulum at E8.5 and is present both in the cytoplasm and nucleus.

\section{METHODS}

Animals. The Yale Institutional Animal Care and Use Committee approved the use of animals in this study.

Plasmid construction and preparation of Hex fusion protein. A fusion protein (GST-Hex) was prepared from the COOH-terminal 76 amino acids of the mouse Hex protein encoded by a full-length cDNA clone Hex1 (3). The cDNA was cloned in frame into the EcoRI site of the GST fusion vector pGEX-5x-3. The eukaryotic expression plasmid pcDNA3 (Invitrogen) was used to make two additional Hex plasmids under the control of the CMV promoter in pcDNA3. One consisted of the full-length Hex cDNA (pCMV-HEX) alone, whereas the second included a FLAG epitope tag at the $3^{\prime}$ end of the cDNA (pCMV-HEXFLAG). The orientation of all the cloned sequences as well as the integrity of the cloning sites was verified by sequencing. pCMV-HEX and pCMVHEXFLAG were used to transiently transfect COS cells. Fusion protein synthesis in transformed bacteria (Escherichia coli DH $5 \alpha$ ) was induced by incubation with $0.5 \mathrm{mM}$ IPTG for $2 \mathrm{~h}$. A total cell lysate was prepared after resuspending the bacterial pellet in 1X PBS and lysed by sonication. After centrifugation at $12,000 \times g$ for $30 \mathrm{~min}$, the supernatant was passed through a GST column (Pharmacia) equilibrated with $1 \mathrm{X}$ PBS and $1 \mathrm{mM}$ PMSF. After washing with PBS and PMSF, the fusion protein was finally eluted in $10 \mathrm{mM}$ glutathione and $50 \mathrm{mM}$ Tris, $\mathrm{pH}$ 8.0.

Antibody production, purification, and Western blotting. Polyclonal antisera to GST-Hex were raised in three rabbits (Charles River Laboratories, Wilmington, MA, U.S.A.) by using standard techniques. After titering, the IgG fraction of Hex antisera was purified on a protein A-sepharose column. The Hex antibody was further purified on a GST-Hex affinity column by use of standard technique (16). Western blotting was performed using standard protocols.

Northern blotting. Total RNA preparation and Northern blotting was carried out following standard procedures.

Immunocytochemistry. After two washes with ice-cold PBS, the COS and MLE15 cells were fixed with $4 \%$ paraformaldehyde in PBS at room temperature for $30 \mathrm{~min}$. They were then permeabilized in PBST for $30 \mathrm{~min}$ at room temperature and blocked in 2\% BSA in PBST for $1 \mathrm{~h}$ at room temperature. Immunostaining of Hex was performed with the rabbit antiHex antibody, and the antibody was detected with a Texas red conjugated secondary antibody $(1: 150)$. Photographs were taken using a Zeiss fluorescence microscope.

Whole mount immunofluorescence. E7.5 and E8.5 mouse embryos fixed in $4 \%$ paraformaldehyde in PBS for $1 \mathrm{~h}$ at room temperature were permeabilized in PBST for $2 \mathrm{~h}$ and blocked with $2 \% \mathrm{BSA}$ in PBST for $2 \mathrm{~h}$ at room temperature. Incubation of anti-Hex antibody (1:200) was performed overnight at $4^{\circ} \mathrm{C}$. After thorough washing with PBST, specific antibody binding was detected by incubation with a Texas red conjugated sec-

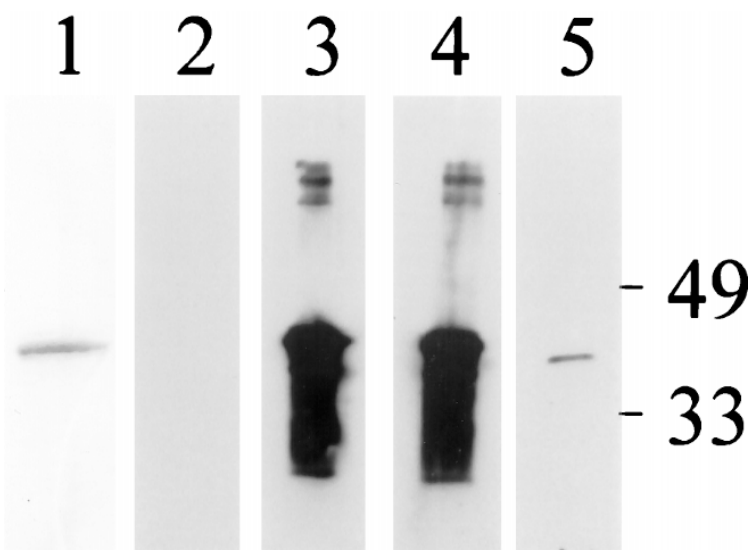

Figure 1. Specificity of anti-Hex antibody. Lane 1, Purified GST-Hex after SDS-PAGE and Coomassie blue staining. Lanes 2-5, Western blots using purified GST-Hex as antigen run on a $10 \%$ polyacrylamide gel. Immunoblot was carried out with preimmune serum (lane 2), Hex antiserum (lane 3), Hex antiserum blocked with purified GST protein (lane 4), or GST-Hex fusion protein (lane 5). See text for details. Numbers used on the right side indicate molecular weights.

ondary antibody $(1: 150)$ at room temperature. Embryos were washed extensively in PBST, mounted in antifade media, and finally observed under a Zeiss laser scanning confocal microscope.

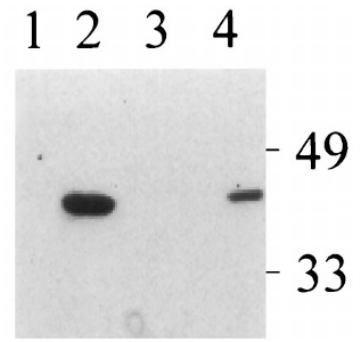

A
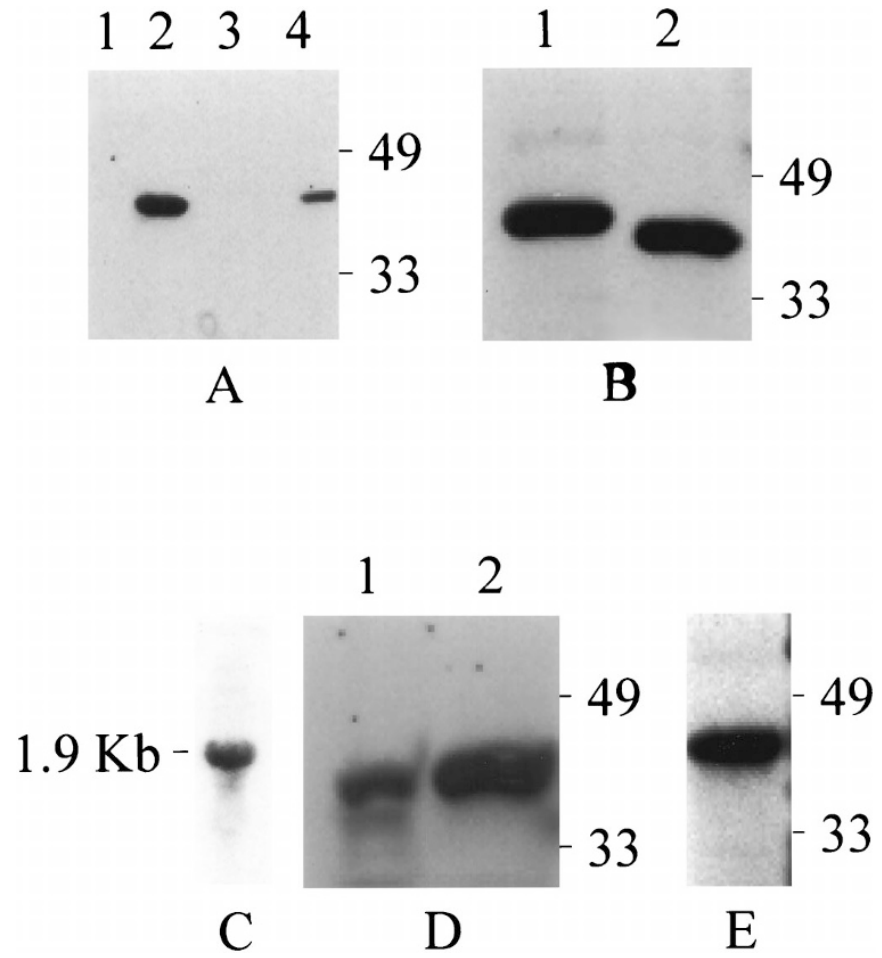

Figure 2. Northern and Western blots of Hex from cultured cells and whole mouse embryos. (A) Equal amounts $(50 \mu \mathrm{g})$ of cytoplasmic (lane 1, 2) and nuclear (lane 3, 4) proteins from nontransfected (lane 1,3) and pCMV-HEX transfected (lane 2, 4) COS cells. (B) Nuclear protein from pCMV-HEXFLAG transfected (lane 1) and pCMV-HEX transfected (lane 2) COS cells. (C) Northern blot of MLE-15 lung epithelial cells showing 1.9-kb Hex mRNA. (D) Nuclear (lane 1) and cytoplasmic (lane 2) protein from nontransfected MLE-15 lung epithelial cells. See text for details. $(E)$ Hex protein in E10.5 whole mouse embryos. For Western blots, $10 \%$ gel was used. Numbers used on the right side indicate molecular weights. 

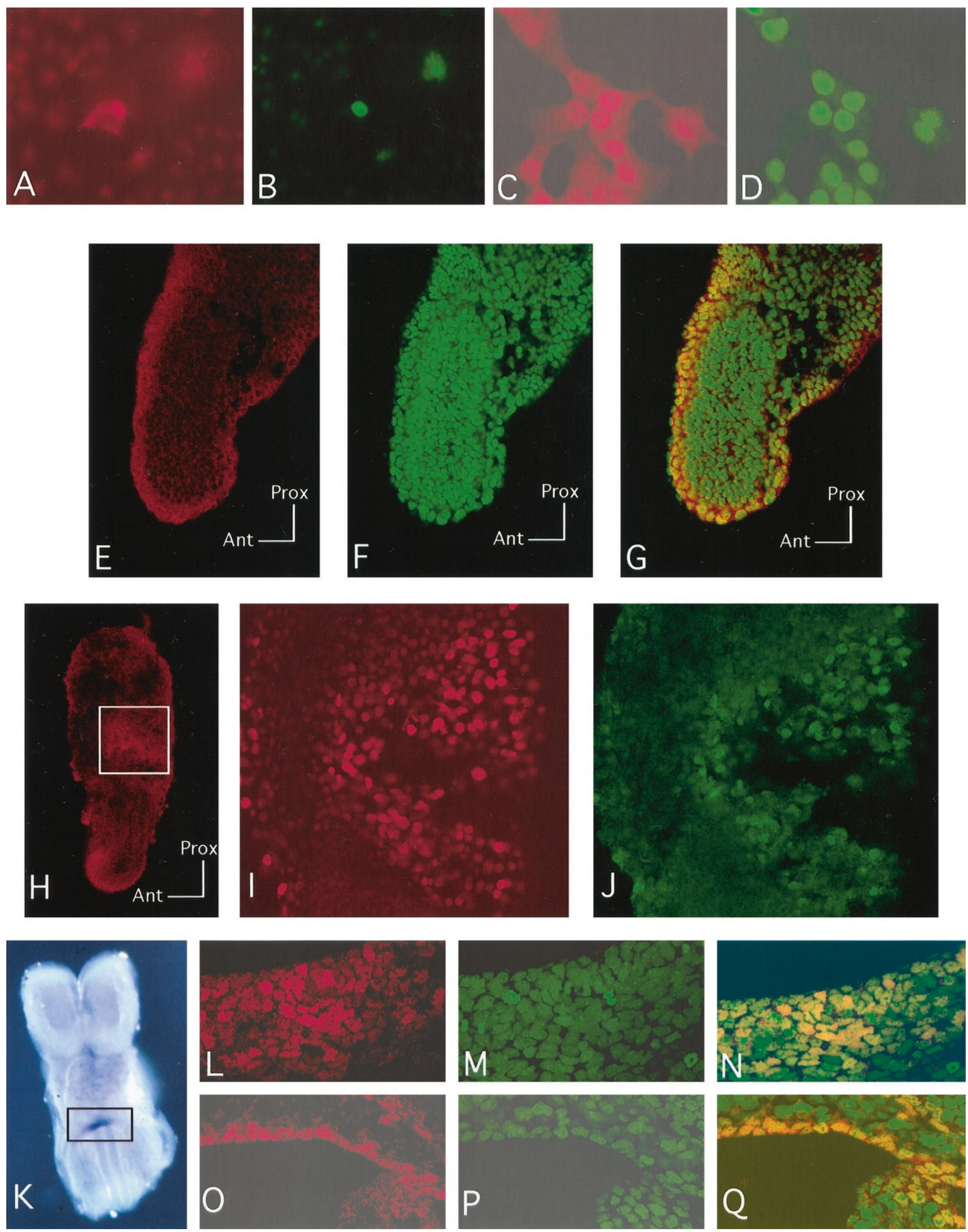

Figure 3. Indirect immunofluorescence showing the distribution of Hex protein in cultured cells $(A-D)$ and mouse embryos $(E-Q)$ stained with anti-Hex antibody or nucleus-specific Sytox green stain. Immunostaining of pCMV-HEX transfected COS cells with anti-Hex antibody $(A)$ and Sytox green counterstain $(B)$. Endogenous expression of Hex protein in MLE-15 cells after immunostaining with anti-Hex antibody $(C)$ and the corresponding nuclear counterstain with Sytox green $(D) .(E)$ Hex-positive definitive endoderm in E7.0 mouse embryo after immunostaining with anti-Hex antibody. Proximal-distal (Prox) and anteroposterior $(A n t)$ axes of the embryo are shown. $(F)$ Counterstain of the same embryo with Sytox green to show the nuclei. $(G)$ Overlay of images $(E)$ and $(F)$ showing that Hex is predominantly cytoplasmic in the definitive endoderm. $(H)$ Immunostaining of developing blood islands $($ boxed) in E7.5 embryo. $(I)$ High-power view of the boxed area in $(H)$ showing nuclear localization of Hex in the developing blood islands. $(J)$ Counterstain of the same region with Sytox 
Whole mount in situ hybridization. Embryos were dissected in PBS and fixed in $4 \%$ paraformaldehyde/PBS for $1 \mathrm{~h}$ at $4^{\circ} \mathrm{C}$. In situ hybridization was performed according to Wilkinson (17). Digoxigenin-labeled antisense Hex RNA probe was used for hybridization.

\section{RESULTS}

Western blot analysis. Rabbits immunized with the GSTHex fusion protein developed a strong immune response as determined by Western blots. To verify the specificity of this response, Western blots were performed against the purified fusion protein after SDS-PAGE and transfer to nitrocellulose (Fig. 1). Figure 1, lane 1 shows the location of the Coomassie blue-stained fusion protein GST-Hex in a gel identical to that from which the transfers were made. Lane 2 demonstrates the lack of reactivity to preimmune sera to the fusion protein. In lane 3, the affinity-purified immune serum strongly labeled the fusion protein as well as some smaller proteins representing proteolytic fragments or contaminating bacterial proteins. To distinguish whether labeling of GST-Hex was entirely due to reactivity with GST epitopes, the immune serum was preabsorbed with purified GST protein, and lane 4 shows that GST-depleted antibody strongly reacted with fusion protein. However, when immune serum was preincubated with GSTHex fusion protein, the antigen-antibody binding was almost abolished (lane 5). These data demonstrate that a significant component of the reactive antibody to GST-Hex was directed against the Hex peptide.

Identification of Hex proteins by immunoblotting in COS cells, MLE 15 cells, and whole mouse embryos. COS cells do not have any endogenous expression of Hex as detected by Western blot. Western blots of COS cells transiently transfected with pCMV-HEX show a $35-\mathrm{kD}$ protein band both in the cytoplasmic and nuclear fractions (Fig. 2A). When COS cells were transfected with pCMV-HEXFLAG, Western blotting detected a $37-\mathrm{kD}$ protein. This size is consistent with the expected size increase due to the FLAG epitope tag (Fig. 2B). Figure $2 D$ shows the presence of $35-\mathrm{kD}$ endogenous Hex protein both in nuclear and cytosolic fractions of the mouse lung epithelial cell line MLE-15. Figure $2 E$ shows the Hex protein in E10.5 whole mouse embryo extract, indicating that the antiserum is specific for mouse tissue.

Identification of Hex protein by immunofluorescence. COS cells were transiently transfected with pCMV-HEX, immunostained with anti-Hex antibody, and counterstained with Sytox green to stain the nuclei (Fig. 3, $A$ and $B$ ). As illustrated in Figure 3, $A$ and $B$, the nucleus and cytoplasm of one transfected COS cell stained positive with Hex antibody. Nontransfected murine lung epithelial MLE-15 cells show endogenous expression of Hex primarily in the nucleus with lower levels in the cytoplasm (Fig. 3, $C$ and $D$ ).
Hex expression in whole mouse embryo. E7.5 and 8.5 embryos were examined for Hex expression by whole mount immunostaining (Fig. 3, $F-K$ ). No signals were detected in E7.5 embryos using preimmune (negative control) sera (data not shown). When staining is performed with anti-Hex antisera, distinct signals are found in the definitive endoderm. Staining in this region, which is predominately cytoplasmic, shows a gradient along the anteroposterior axis with the highest intensity on the anterior side of the embryos (Fig. $3 F$ ). Hex is also expressed in the developing blood islands of the extraembryonic region at this stage (Fig. 3, $G$ and $H$ ). However, in these cells, Hex immunoreactivity is confined solely to the nuclei of cells (Fig. 3H). At E8.5, strong staining is found in the hepatic diverticulum, the region of the foregut that forms the liver (Fig. 3I). In the foregut endodermal cells that are most anterior and form the leading edge of endodermal cells that are migrating into the precardiac mesoderm, staining is quite intense and is predominately nuclear. However, cells in the region that are more posterior and, therefore, more distant from the precardiac mesoderm express lower levels of Hex, and the expression is primarily cytoplasmic rather than nuclear (Fig. $3 K)$.

\section{DISCUSSION}

Hex is one of the earliest markers of the anterior aspect of the embryo, suggesting a role in anteroposterior patterning (5, 18). In addition, Hex is expressed in several organs derived from the foregut and is seen in early hematopoietic and endothelial cells (3). The conservation of Hex in a diverse group of vertebrates including mouse, rat, chick, frog, and human (3, 4, $14,19,20)$ strongly suggests that Hex plays a fundamental role in early embryonic development. The present study is the first to examine Hex protein expression at the subcellular level in cultured cells as well as in the developing mouse embryo. We show that the Hex protein, which functions as a DNA-binding transcription factor, is found both in the nucleus and cytoplasm of both transfected and nontransfected COS and MLE-15 cells and in mouse embryos in vivo. This suggests that one important mechanism for regulating the transcriptional activity of Hex is the regulation of its nuclear-cytoplasmic localization.

The immunochemical characterization of Hex as a $35-\mathrm{kD}$ polypeptide is consistent with a molecular weight of $31 \mathrm{kD}$ as predicted from its amino acid sequence. The Western blot and immunofluorescence studies indicate that Hex is localized in both the nucleus and cytoplasm of Hex-transfected COS cells as well as nontransfected MLE-15 lung epithelial cell, which express Hex endogenously as evidenced by the presence of Hex mRNA in this cell line (Fig. 2C). The distribution of the Hex protein in developing mouse embryos correlates well with its mRNA distribution as determined by whole-mount in situ hybridization (5). Distinct signals are found in the definitive

green. $(K)$ In situ hybridization of E8.5 embryo with full-length Hex antisense RNA showing Hex expression in the hepatic diverticulum (boxed). Immunostaining of the hepatic diverticulum (boxed in $K$ ) with anti-Hex antibody at higher magnification $(L)$ and counterstained with Sytox green $(M)$. ( $N$ ) Overlay of images $(L)$ and $(M)$ shows the nuclear localization of Hex in this region. Low-power view of the hepatic diverticulum, immunostained with anti-Hex antibody $(O)$ counterstained with Sytox green $(P)$ and their overlay $(Q)$. Note that Hex signal is predominantly cytoplasmic in the foregut endoderm lateral to the hepatic diverticulum. Original magnification: $A$ and $B, \times 40 ; C$ and $D, \times 100 ; E-H, \times 10 ; I$ and $J, \times 40 ; K, \times 5 ; L-N, \times 60 ; O-Q, \times 40$. 
endoderm of E7.5 embryos in an asymmetric pattern: Hex is found predominantly on the anterior side of the embryo, and this signal is cytoplasmic. In contrast, the signal seen in the developing blood islands is localized in the nuclei. Approximately $1 \mathrm{~d}$ later, Hex expression in the region of developing liver is both nuclear and cytoplasmic. It appears that the foregut endodermal cells that are closest to the precardiac mesoderm express high levels of Hex that is predominately nuclear in localization, and cells on the posterior aspect of the foregut in this same region express Hex at much lower levels and mainly in the cytoplasm. The functional significance of this differential intracellular localization of Hex protein is not clear, but is likely to be involved in regulating its function as a transcription factor as has previously been demonstrated with Antennapedia, Prospero, and Exd proteins in Drosophila development (21-25) and Csx in mice (10).

Our results suggest that Hex localization in either the nucleus or cytoplasm is a potential mechanism by which its function as a transcription factor is regulated. It is likely that shuttling of Hex between the nucleus and cytoplasm is mediated through an NLS $(10,26)$ and/or an NES $(27,28)$, both of which have been found in the homeodomains of other homeobox proteins. Hex does not contain a typical nuclear localization signal like other transcription factors, but a computer search reveals the presence of three categories of potential NLS (pat4, pat7, and bipartite) in its homeodomain (29). Hex also shows a strong homology at the amino acid level with the NES region of other homeobox proteins including chick engrailed, mouse HOXC8, and chick HOXB4 (28). Studies are in progress to see if any of these potential NLS or NES sequences are functional in Hex and are responsible for its nucleocytoplasmic transport. Additionally, some proteins require posttranslational modification such as phosphorylation, glycosylation, or ubiquitination for their nuclear translocation (30-32). It remains to be seen whether Hex also requires similar modification at the posttranslational level for its nucleocytoplasmic transport.

In summary, we find that mouse Hex is a soluble protein with an apparent molecular mass of $35-37 \mathrm{kD}$ and is expressed both in nuclei and cytoplasm of cultured cells as evidenced by Western blot and immunofluorescence. At E7.5, Hex protein is localized in the cytoplasm of definitive endoderm in a gradient along the anteroposterior axis and is seen in the nuclei of the cells in the region of the developing blood islands. Hex is also expressed in both the cytoplasm and the nuclei of the cells in the hepatic diverticulum at the E8.5. Further immunochemical studies are needed to define the distribution of Hex protein during development. Similarly, it is important to determine the functional significance of the nuclear/cytoplasmic compartmentalization that we have here demonstrated.

\section{REFERENCES}

1. Gehring WJ 1987 Homeo boxes in the study of development. Science 236:1245-1252

2. Duboule D 1994 Guidebook to the Homeobox Genes. Oxford University Press, New York
3. Bedford FK, Ashworth A, Enver T, Wiedemann LM 1993 HEX: a novel homeobox gene expressed during haematopoiesis and conserved between mouse and human. Nucleic Acids Res 21:1245-1249

4. Crompton MR, Bartlett TJ, MacGregor AD, Manfioletti G, Buratti E, Giancotti V, Goodwin GH 1992 Identification of a novel vertebrate homeobox gene expressed in haematopoietic cells. Nucleic Acids Res 20:5661-5667

5. Thomas PQ, Brown A, Beddington RS 1998 Hex: a homeobox gene revealing peri-implantation asymmetry in the mouse embryo and an early transient marker of endothelial cell precursors. Development 125:85-94

6. Keng VW, Fujimori KE, Myint Z, Tamamaki N, Nojyo Y, Noguchi T 1998 Expression of Hex mRNA in early murine postimplantation embryo development. FEBS Lett 426:183-186

7. Corsetti MT, Levi G, Lancia F, Sanseverino L, Ferrini S, Boncinelli E, Corte G 1995 Nuclear localization of three Hox homeoproteins. J Cell Sci 108:187-193

8. Driever W, Nusslein-Volhard C 1988 A gradient of bicoid protein in Drosophila embryos. Cell 54:83-93

9. Mlodzik M, Gehring WJ 1987 Expression of the caudal gene in the germ line of Drosophila: formation of an RNA and protein gradient during early embryogenesis. Cell 48:465-478

10. Kasahara H, Bartunkova S, Schinke M, Tanaka M, Izumo S 1998 Cardiac and extracardiac expression of Csx/Nkx2.5 homeodomain protein. Circ Res 82:936-946

11. Aspland SE, White RAH 1997 Nucleocytoplasmic localisation of extradenticle protein is spatially regulated throughout development in Drosophila. Development 124:741-747

12. Mann RS, Abu-Shaar M 1996 Nuclear import of the homeodomain protein extradenticle in response to Wg and Dpp signalling. Nature 383:630-633

13. Joliot A, Trembleau A, Raposo G, Calvet S, Volovitch M, Prochiantz A 1997 Association of engrailed homeoproteins with vesicles presenting caveolae-like properties. Development 124:1865-1875

14. Newman CS, Chia F, Krieg PA 1997 The XHex homeobox gene is expressed during development of the vascular endothelium: overexpression leads to an increase in vascular endothelial cell number. Mech Dev 66:83-93

15. Bogue CW, Ganea GR, Sturn E, Ianucci R, Jacobs HC 2000 Hex expression suggests a role in the development and function of organs derived from foregut endoderm. Dev Dyn 219:84-89

16. Harlow E, Lane D 1988 Antibodies: A Laboratory Manual. Cold Spring Harbor Laboratory, Cold Spring Harbor, NY

17. Wilkinson DG 1992 Whole mount in situ hybridization of vertebrate embryos. In: Wilkinson DG (ed) In Situ Hybridization: A Practical Approach, Vol 109. IRL Press, Oxford, UK, pp 75-83

18. Beddington RS, Robertson EJ 1999 Axis development and early asymmetry in mammals. Cell 96:195-209

19. Hromas R, Radich J, Collins S 1993 PCR cloning of an orphan homeobox gene (PRH) preferentially expressed in myeloid and liver cells. Biochem Biophys Res Commun 195:976-983

20. Tanaka T, Inazu T, Yamada K, Myint Z, Keng VW, Inoue Y, Taniguchi N, Noguchi T 1999 cDNA cloning and expression of rat homeobox gene, Hex, and functional characterization of the protein. Biochem J 339:111-117

21. Hirata J, Nakagoshi H, Nabeshima Y, Matsuzaki F 1995 Asymmetric segregation of the homeodomain protein Prospero during Drosophila development. Nature 377:627630

22. Hassan B, Li L, Bremer KA, Chang W, Pinsonneault J, Vaessin H 1997 Prospero is a panneural transcription factor that modulates homeodomain protein activity. Proc Natl Acad Sci USA 94:10991-10996

23. Casares F, Mann RS 1998 Control of antennal versus leg development in Drosophila Nature 392:723-726

24. Mercader N, Leonardo E, Azpiazu N, Serrano A, Morata G, Martinez C, Torres M 1999 Conserved regulation of proximodistal limb axis development by Meis 1/Hth. Nature 402:425-429

25. Azpiazu N, Morata G 1998 Functional and regulatory interactions between Hox and extradenticle genes. Genes Dev 12:261-273

26. Moede T, Leibiger B, Pour HG, Berggren P, Leibiger IB 1999 Identification of a nuclear localization signal, RRMKWKK, in the homeodomain transcription factor PDX-1. FEBS Lett 461:229-234

27. Gorlich D, Mattaj IW 1996 Nucleocytoplasmic transport. Science 271:1513-1518

28. Maizel A, Bensaude O, Prochiantz A, Joliot A 1999 A short region of its homeodomain is necessary for engrailed nuclear export and secretion. Development 126:31833190

29. Hicks GR, Raikhel NV 1995 Protein import into the nucleus: an integrated view. Annu Rev Cell Dev Biol 11:155-188

30. Matunis MJ, Coutavas E, Blobel G 1996 A novel ubiquitin-like modification modulates the partitioning of the Ran-GTPase-activating protein RanGAP1 between the cytosol and the nuclear pore complex. J Cell Biol 135:1457-1470

31. Macfarlane WM, McKinnon CM, Felton-Edkins ZA, Cragg H, James RF, Docherty K 1999 Glucose stimulates translocation of the homeodomain transcription factor PDX1 from the cytoplasm to the nucleus in pancreatic beta-cells. J Biol Chem 274:1011-1016

32. Duverger E, Pellerin-Mendes C, Mayer R, Roche AC, Monsigny M 1995 Nuclear import of glycoconjugates is distinct from the classical NLS pathway. J Cell Sci 108:1325-1332 